\title{
The Merton Structural Model and IRB Compliance
}

\author{
Matej Jovan ${ }^{1}$
}

\begin{abstract}
This paper discusses the 1974 Merton's model in light of the minimum regulatory requirements of the Internal Ratings-Based (IRB) Approach provided in the Directive 2006/48/EC of the European Parliament and of the Council for the calculation of capital requirement for credit risk. The basic purpose is to illustrate potential deficiencies of the model in assigning obligors ratings and/or estimating probability of default to which supervisors should be attentive when validating this model in bank's IRB approach. The procedures of three estimation methods of Merton's model are described (calibration, Moody's KMV, maximum likelihood estimation), based on which deficiencies of this model can be identified. The Merton's model per se does not ensure compliance with the minimum requirements of the IRB approach for the estimation of probability of default, as its theoretical assumptions often do not reflect reality. It is therefore necessary to calibrate the fundamental parameters estimated by the model using empirical data on defaults, which must be defined in accordance with the regulatory minimum requirements, and must be representative of the population for which the model is valid. Results on the simulated data also show that calibration method provides different estimates of probability of default for the same obligors compared to other two methods. Differences are mainly influenced by the volatility of equity and leverage in the time series, which calibration method does not sufficiently account for. Some regulatory minimum requirements can be relaxed when obligors are being assigned ratings on the basis of the Merton's model estimation methods. However, the results of the analysis on simulated and empirical data show that different estimation methods generate different obligor credit rating assignments.
\end{abstract}

\section{Introduction}

With the advent of the Internal Ratings-Based (IRB) Approach in the Directive 2006/ 48/EC of the European Parliament and of the Council (CRD), statistical modeling has come to the fore of credit risk management. Not only are default prediction models the centre of interest when it comes to the IRB approach, credit models also form the basis of the equation for calculating capital requirements for credit risk using an IRB approach (Gordy, 2003; Basel Committee on Banking Supervision, 2004).

From a quantitative point of view, the IRB approach is defined by three random variables as key risk parameters: Probability-of-Default (PD), Loss-Given-Default and Conversion Factor. These parameters are always input data for calculating capital requirements for credit risk using the IRB approach. The best investigated of them is PD, which

\footnotetext{
${ }^{1}$ Bank of Slovenia, Banking Supervision Department, Ljubljana, Slovenia; matej.jovan@ bsi.si
} 
therefore has the greatest number of methodologies for its estimation. The CRD takes this methodological diversity into consideration, and does not prescribe any methodology that a priori would be more desirable than any other for rating obligors and estimating PD. European banking supervisors within the Committee of European Banking Supervisors in Guidelines on the implementation, validation and assessment of Advanced Measurement (AMA) and Internal Ratings Based (IRB) Approaches (2006, hereafter Guidelines on Validation) also came to the same conclusions, and explicitly stated that the use of multiple methodologies is allowed, and that it is up to banks to substantiate their models if they intend to use them to calculate capital requirements.

The Oesterreichische Nationalbank in the document Rating models and Validation (2005) classifies the existing methodologies for estimating PD into three groups: (1) heuristic models, (2) statistical models, and (3) causal models. This paper only focus on the third type of models, more specifically the structural model first articulated by Merton (1974), which Altman (2006) calls a first-generation structural model. On the basis of the Merton structural model estimates of PD are made for fictitious companies using simulated data, and for certain Slovenian companies in the main Ljubljana stock exchange index (SBI20). Estimates of PD are provided for a one-year time horizon, which are derived from the movement of market prices of equity. To this end, three methods for estimating PD will be used, all based on the 1974 Merton model: (1) the calibration method (e.g. see Bruche, 2005), (2) Moodys KMV (the MKMV, see Crosbie and Bohn, 2003) method, and the maximum likelihood estimation (MLE) technique proposed by Duan $(1994,2004)$. The purpose of this paper is to identify any differences in the estimates of parameters under individual methods, and to establish the usefulness of the traditional Merton's model in the IRB approach, in general and for listed Slovenian companies, from the point of view of rating obligors or from the point of view of estimating PD.

This paper is organized as follows: the second section provides the theory of the 1974 Merton's model, which represents the basis for forecasting PD. The third section describes the derivation of the calculation of asset value and asset volatility on the basis of empirical (market) value of equity and estimates of their volatility. The fourth section illustrates the point of view of meeting the minimum requirements of the IRB approach, depending on whether the model is being used for the purpose of rating obligors or for the purpose of estimating PD. The fifth section presents the data. The sixth section provides calculations of one-year estimates of PD under all three methods. The seventh section summarizes the papers findings, and gives the starting points for further discussion.

\section{Assumptions of the structural model}

In the 1974 Merton's model, equity represents a call option on the companys assets held by the holders of the equity. The financing of the company is simple. It consists of one type of equity issued at time $t\left(E_{t}\right)$ and zero-coupon debt issued at $t\left(D_{t}\right)$ with face value of $L$ maturing at time $T$. The strike price of the call option is the same as $L$. $L$ already includes some accrued interest at a rate reflecting the company's riskiness. Debt holders finance company's assets at time $t$ with an amount equal to $D_{t}$, and at $T$ they receive an amount equal to $\min \left[A_{T}, L\right]$, where $A_{T}$ is the market value of assets at maturity. In this 
financing structure the market value of the companys assets at time $t$ is given by:

$$
A_{t}=E_{t}+D_{t} .
$$

The dynamic of asset value $A_{t}$ follows a geometric Brownian motion as follows:

$$
d \ln A_{t}=\mu_{A} d t+\sigma_{A} d Z_{t},
$$

where $\mu_{A}$ is the constant return or drift, $\sigma_{A}$ is the constant standard deviation, and $Z_{t}$ is the normal random variable $N \sim(0,1)$. The solution to equation (2.2) can be obtained using Ito's lemma, and is

$$
A_{T}=A_{t} e^{\left(\mu_{A}-\frac{\sigma_{A}^{2}}{2}\right)(T-t)+\sigma_{A} \sqrt{(T-t)} Z_{t}},
$$

where $(T-t)$ is remaining maturity.

The market value of the assets at time $t$ has a log-normal distribution. The natural logarithm of returns on the companys assets $\ln \left(A_{T} / A_{t}\right)$ is distributed normally

$$
N \sim\left(\left(\mu_{A}-\frac{\sigma_{A}^{2}}{2}\right)(T-t), \sigma_{A}^{2}(T-t)\right) .
$$

The market value of the companys equity at time $T$ is given by

$$
E_{T}=\max \left[A_{T}-L, 0\right] .
$$

Equity holders will exercise the option if the market value of the assets at maturity $\left(A_{T}\right)$ is higher than the strike price of the option. In this event they will purchase the companys assets at a price below the market value. In the event $A_{T}<L$, which defines the default event in Merton's framework, there are no assets left that could be taken over by the equity holders.

Under certain assumptions (Merton, 1974) the solution to (2.4) for equity values in $t$ is given by the Black-Scholes (1973) equation for pricing a call option

$$
E_{t}=A_{t} \Phi\left(d_{1}\right)-L e^{-r(T-t)} \Phi\left(d_{2}\right),
$$

where

$$
\begin{aligned}
& d_{1}= \frac{\ln \left(\frac{A_{t}}{L}\right)+\left(r+\frac{\sigma_{A}^{2}}{2}\right)(T-t)}{\sigma_{A} \sqrt{(T-t)}}, \\
& d_{2}=\frac{\ln \left(\frac{A_{t}}{L}\right)+\left(r-\frac{\sigma_{A}^{2}}{2}\right)(T-t)}{\sigma_{A} \sqrt{(T-t)}},
\end{aligned}
$$

and $r$ is the risk-free interest rate, and $\Phi()$ is the cumulative distribution function of the standard normal variable. Equation (2.7) describes the number of standard deviations of natural logarithms of $A_{t} / L$ from the mean. MKMV calls this interval the distance-todefault, but it is defined differently elsewhere (see Crosbie and Bohn, 2003). 
The model so defined, the traditional Merton's model, forms the basis for estimating PD.

By rearranging (2.3) an estimate of $\mathrm{PD}$ is

$$
\begin{aligned}
P D_{t} & =\mathbb{P}\left[A_{T} \leq L\right] \\
& =\mathbb{P}\left[\ln \left(A_{t}\right)+\left(\mu_{A}-\frac{\sigma_{A}^{2}}{2}\right)(T-t)+\sigma_{A} \sqrt{(T-t)} Z_{t} \leq \ln (L)\right] \\
& =\mathbb{P}\left[Z_{t} \leq-\frac{\ln \left(\frac{A_{t}}{L}\right)+\left(\mu_{A}-\frac{\sigma_{A}^{2}}{2}\right)(T-t)}{\sigma_{A} \sqrt{(T-t)}}\right] \\
& =\int_{-\infty}^{-\frac{\ln \left(\frac{A_{t}}{L}\right)+\left(\mu_{A}-\frac{\sigma_{A}^{2}}{2}\right)(T-t)}{\sigma_{A} \sqrt{(T-t)}}} \phi(x) d x .
\end{aligned}
$$

Here, $\phi$ is the probability density function of a standard normal variable. Note that equation (2.7) is not a function of $\mu_{A}$, like (2.8). If $\mu_{A}=r$, then PD is identical in both equations.

\section{Market value of assets from market value of equity}

The market value of assets $A_{t}$ is a random variable that cannot be observed directly. It would therefore also be impossible to directly estimate the drift and the standard deviation in the movement of the natural logarithms of returns on the market value of assets. All three parameters are necessary for the estimation of a company's PD. Notwithstanding that the traditional Merton's model assumes a simple debt structure, and is thus highly simplified, it proceeds from an assumption of the observed market values of assets that empirically cannot be realized. However, the market value of assets, the drift and the standard deviation can be estimated indirectly from (2.5) and the observed market value of equity $E_{t}$.

This paper makes use of three methods for estimating these three parameters, from which the one-year PD is estimated. All three methods are implemented in the $R$ statistical software ${ }^{2}$.

\subsection{Calibration method}

The first method, which Bruche (2005) named the calibration method, uses iteration to solve the system of two equations with two unknowns (Bruche, 2005; Crosbie and Bohn, 2003; Bluhm et al., 2003; Ericsson and Reneby, 2005; Elizade, 2005), namely (2.5) and

$$
\sigma_{E}=\frac{A_{t}}{E_{t}} \Phi\left(d_{2}\right) \sigma_{A}
$$

\footnotetext{
${ }^{2} \mathrm{R}$ is freely accessible at http://www.r-project.org.
} 
This first method is defined as follows. The initial values $\hat{A}_{t}^{(i)}$ and $\hat{\sigma}_{A}^{(i)}$ are chosen arbitrarily ${ }^{3}$. Initial values of $\hat{A}_{0}^{(1)}=E_{0}$ and $\hat{\sigma}_{A}^{(1)}=\sigma_{E}$ were used in the paper. For empirical data, $E_{0}$ is the market capitalisation of the company at the end of the year (number of shares multiplied by share price). For simulated data, $E_{0}$ is defined in section 5.1. For empirical data, $\sigma_{E}$ is estimated from the time series of daily natural logarithms of returns on the companys equity

$$
\begin{aligned}
\hat{\sigma}_{E} & =\sqrt{\frac{1}{n} \sum_{t=1}^{n}\left(X_{t}-\bar{X}\right)^{2}} \sqrt{250}, \\
\bar{X} & =\frac{1}{n} \sum_{t=1}^{n} X_{t}
\end{aligned}
$$

where $X_{t} \equiv \ln \left(E_{t} / E_{t-1}\right), t=(0, \ldots, n)$ and $r$ is the interest rate on 12-month treasury bills. For simulated data, $\sigma_{E}$ is defined in section 5.1. For empirical data, $L$ is the total book liabilities from the consolidated balance sheet at the end of the year. For simulated data, $L$ is defined in section 5.1. The defined values are input into the following iterative procedure:

1. (2.5) is solved;

2. the new solution of $\hat{A}_{0}^{(i+1)}$ is input into (3.1);

3. the new solutions of $\hat{A}_{0}^{(i+1)}$ and $\hat{\sigma}_{A}^{(i+1)}$ are re-input into (2.5).

The procedure is repeated until the differences in $\hat{A}_{0}$ and $\hat{\sigma}_{A}$ between successive iterations are sufficiently small ${ }^{4}$. Usually it only takes a small number of iterations for the values to converge to produce estimates of the desired parameters. The values obtained allow for the calculation of an estimate of PD from (2.8). For this estimate of PD, $\mu_{A}=r$ and the one-year return on the companys assets is equal to the return on 12-month treasury bills 5 .

\subsection{MKMV method}

The second iterative procedure estimates $A_{t}, \sigma_{A}$ and $\mu_{A}$. The latter is important for the estimation of PD as a function of drift. The second iterative procedure follows the disclosed part of the MKMV methodology for the calculation of Expected Default Frequency (Crosbie and Bohn, 2003; Vassalou and Xing, 2004; Duffie et al., 2005; Duan et al., 2004). The time series of daily market value of equity from which the parameter is estimated is equal to $n$ days, where $t=(0, \ldots, n)$. Following the same notation as Duan et al. (2004), the coefficient $h=1 /(n /$ no. of years $)$ is introduced, which serves to convert the daily values into annual values of the parameters. The initial values entered into (2.5) are $\hat{A}_{t h}^{(1)}=E_{t h}$,

\footnotetext{
${ }^{3} i^{t h}$ itertion, $i=1$ is the first in the series of iterations, $t=0$ and $T-t=1$.

${ }^{4}$ An absolute difference of $10^{-10}$ is used in this paper.

${ }^{5}$ The treasury bill yield is commonly used in the literature as the risk-free interest rate $r$.
} 
while $L$ and $r$ are defined as in the first iterative method, $\hat{\mu}_{A}$ and $\hat{\sigma}_{A}$ are stipulated arbitrarily, and the maximum maturity of the debt $L$ is equal to $T$. Each iteration produces a time series of daily values $\hat{A}_{t h}$, where the maturity of the debt ranges from $1 \leq(T-t h) \leq T$. The procedure is as follows:

1. calculation of the daily value of $\hat{A}_{t h}^{(i)}, t h=(0, \ldots, n h)$ from $(2.5)$;

2. calculation of the arithmetic mean of the sample

$$
\begin{aligned}
\bar{R}^{(i)} & =\frac{1}{n} \sum_{t=1}^{n} \hat{R}_{t}^{(i)} \\
\hat{R}_{t}^{(i)} & \equiv \ln \left(\hat{A}_{t h}^{(i)} / \hat{A}_{(t-1) h}^{(i)}\right) ;
\end{aligned}
$$

3. calculation of the standard deviation

$$
\hat{\sigma}_{A}^{(i+1)}=\sqrt{\frac{1}{n h} \sum_{t=1}^{n}\left(\hat{R}_{t}-\bar{R}\right)^{2}} \text {; }
$$

4. calculation of the drift $\hat{\mu}_{A}$

$$
\hat{\mu}_{A}^{(i+1)}=\bar{R}^{(i)} \frac{1}{h}+\frac{\hat{\sigma}_{A}^{2(i+1)}}{2}
$$

5. return to the first step for the calculation of $\hat{A}_{t h}^{(i+1)}$ using $\hat{\mu}_{A}^{(i+1)}$ and $\hat{\sigma}_{A}^{(i+1)}$ from the second step.

The procedure is repeated until the differences in $\mu_{A}$ and $\sigma_{A}$ between successive iterations are sufficiently small. The procedure usually converges quickly. The values obtained allow for the calculation of a one-year estimate of PD from (2.8). This time the estimate of PD is the function $\mu_{A}$, and the one-year return on the companys assets is equal to the average return on the company in the previous sampling period.

For the given sample it is possible to use an analytical solution for the standard errors in $\mu_{A}$ and $\sigma_{A}$, respectively, as follows:

$$
\begin{aligned}
& \text { s.e. }\left(\hat{\mu}_{A}\right)=\text { s.e. }\left(\bar{R}+\frac{\hat{\sigma}_{A}^{2}}{2}\right) \frac{1}{h}=\frac{\hat{\sigma}_{A}}{\sqrt{n} h} \\
& \text { s.e. }\left(\hat{\sigma}_{A}\right)=\frac{\hat{\sigma}_{A}}{\sqrt{2 n} h} .
\end{aligned}
$$

\subsection{MLE - Maximum likelihood estimation method}

The third method follows the methodology proposed by Duan (1994), later augmented by Duan et al. (2003, 2004). This is an estimation of parameters based on maximum likelihood estimation. 
Duan et al. (2004) introduces the following log-likelihood equation for the estimation of $\mu_{A}, \sigma_{A}$, and $A_{t h}$ on the basis of observed market values of equity

$l\left(\hat{\theta}_{A} ; \hat{A}_{t h} \mid E_{t h}\right)=-\frac{n}{2} \ln \left(2 \pi \hat{\sigma}_{A}^{2} h\right)-\frac{1}{2} \sum_{t=1}^{n} \frac{\left(\hat{R}_{t}-\left(\hat{\mu}_{A}-\frac{\hat{\sigma}_{A}^{2}}{2}\right) h\right)^{2}}{\hat{\sigma}_{A}^{2} h}-\sum_{t=0}^{n} \ln \left(\hat{A}_{t h}\right)-\sum_{t=0}^{n} \ln \left(\Phi\left(d_{1}\right)\right)$

where $\hat{\theta}_{A} \equiv\left(\hat{\mu}_{A}, \hat{\sigma}_{A}\right), \hat{A}_{t h}$ is estimated from (2.5) and $\Phi$ is the cumulative distribution function of a standard normal variable.

The time series of daily market value of equity from which the parameter is estimated is equal to $n$ days, where $t=(0, \ldots, n h)$. Each iteration produces a time series of daily values $\hat{A}_{t h}$, where the maturity of the debt ranges over $1 \leq(T-t h) \leq T$. All the initial values input into (2.5) are the same as in the MKMV method.

The estimation procedure is as follows:

1. calculation of the daily values of $\hat{A}_{t h}^{(i)}, t h=(0, \ldots, n h)$ from $(2.5)$;

2. calculation of $\hat{\mu}_{A}^{(i+1)}, \hat{\sigma}_{A}^{(i+1)}$ and their standard errors by finding the maximum (3.7). The standard errors are derived from a diagonal of the Hessian matrix ${ }^{6}$;

3. return to the first step for the calculation of $\hat{A}_{t h}^{(i+1)}$ using $\hat{\mu}_{A}^{(i+1)}$ and $\hat{\sigma}_{A}^{(i+1)}$ from the second step.

The second step involves the iterative solution of the optimization problem, and thus an iterative procedure for finding the maximum of the logarithm of the maximum likelihood function within the second iterative procedure consisting of the steps described. Duan et al. (2004) show that the above iterative procedure is an expectation-maximization algorithm. They find that the MKMV method is equivalent to the MLE method, but state that the latter method is preferable for inference statistics. The equivalence can be observed both in the equivalent estimates of the parameters and in the logic of the expectation-maximization algorithm. The MLE method should also produce equivalent standard errors for $\mu_{A}$ and $\sigma_{A}$ as in (3.5) and (3.6). This can be observed in the analytical solution to the log-likelihood equation for estimating standard errors for $\mu_{A}$ and $\sigma_{A}$, in the case of a univariate standard normal random variable

$$
l\left(\bar{R}, \hat{\sigma}_{A} ; \hat{R}_{t}\right)=-\frac{n}{2} \ln \left(2 \pi \hat{\sigma}_{A}^{2} h\right)-\frac{1}{2} \sum_{t=1}^{n} \frac{\left(\hat{R}_{t}-\bar{R} h\right)^{2}}{\hat{\sigma}_{A}^{2} h} .
$$

Equations (3.2) and (3.3) are both analytical solutions to log-likelihood equation (3.8) for $\bar{R}$ and $\hat{\sigma}_{A}{ }^{7}$ or $\hat{\mu}_{A}$. This completes the evidence of the equivalence of the MKMV and MLE methods.

\footnotetext{
${ }^{6}$ In this paper the optim (Nelder-Mead) algorithm was used in $R$ statistical software and the diagonal of the Hessian matrix was used from it.

${ }^{7}$ The proof is in Campbell, Lo and MacKinlay (1996), chapter 9.
} 


\section{Compliance of the Merton's model with the minimum requirements of the IRB approach}

With the advent of the CRD, banks are be able to use a variety of rating systems for assigning obligors to an obligor rating scale and for estimating PD. The Merton's model and its variants (the MKMV) seem to be the most suitable for listed companies. However, even a theoretically clear model like the Merton's model, and the various methods for estimating the parameters of the model, are subject to the minimum requirements of the CRD, which the model must fulfill if its results are to be used to calculate capital requirements for credit risk. The theoretical model per se does not fulfill the minimum requirements.

In order to analyze compliance with the minimum requirements, it is reasonable to observe the Merton's model separately for each of its two purposes, namely as a method for assigning obligors to an obligor rating scale, and as a method for estimating PD. The latter can implicitly be considered as an assignment to a continuous rating scale or direct assignment of rating grades, as provided for by the CRD (Annex VII, Part 4, paragraph 4). In this case it is a method for assigning and estimating PD.

\subsection{Compliance of the definition of default}

The following definition of default is determined in the CRD:

"A 'default' shall be considered to have occurred with regard to a particular obligor when either or both of the two following events has taken place:

(a) the credit institution considers that the obligor is unlikely to pay its credit obligations to the credit institution, the parent undertaking or any of its subsidiaries in full, without recourse by the credit institution to actions such as realising security (if held);

(b) the obligor is past due more than 90 days on any material credit obligation to the credit institution, the parent undertaking or any of its subsidiaries" (paragraph 44, Part 4, Annex VII of the CRD).

In the Merton's model a default is considered to have occurred when the value of the debt $L$ exceeds the market value of the assets at maturity. This situation could be interpreted in a way that the event (a) in the above definition of default has taken place, i.e. the obligor is unlikely to pay its credit obligations (at maturity). However, the definition of default of the Merton's model is not in full compliance with the definition in the CRD, as it does not cover all potential events included in the CRD's definition of default.

Another (possible) non-compliance of Mertons model regarding definition of default is the restriction of default time to the maturity of the debt. Default can be defined only at the maturity of debt (e.g. one-year) and not earlier, meaning that the estimates of PD are valid for precisely that time point. However, CRD stipulates that "probability of default means the probability of default of a counterparty over a one year period" (paragraph 25, Article 4). Rigorous interpretation of this definition in CRD might put to the question the compliance of PD estimates from Merton's model. To the author's knowledge, in the IRB approval processes there were no rejections by the supervisors of Merton's model because of the restriction of default time to the maturity of debt.

The composition of the debt represented by the value $L$ is closely tied to the definition 
of default. The value of the debt $L$ can be interpreted as a calibrant, i.e. the value that can be calibrated with regard to empirical data on defaults that were preliminarily defined in accordance with the CRD. In other words, for a compliant definition of default, the value of the debt $L$ is the value that must be calibrated to reflect the appropriate debt structure in connection with empirical data on defaults. Davydenko (2005) analyses this relationship, but the definitions of default from which the values of the debt $L$ are calibrated are not fully in accordance with the definitions in the CRD. When assessing Expected Default Frequency, the MKMV method includes the companys short-term debt and half of its long-term debt in $L$, which is an industry benchmark.

The distance-to-default can also be interpreted as a calibrant. Using this type of calibration to empirical data on defaults, MKMV (Crosbie and Bohn, 2003) for example addresses the problem of inaccurate calibration of the structure of $L$ and all other influences not covered. The calibration of distance-to-default implicitly calibrates all the estimated parameters.

As in Bharath and Shumway (2008), this paper uses the total book liabilities as the value $L$ for empirical data.

Where a bank is using the Merton's model or variant estimation methods in the IRB approach merely as a method for assigning obligors to an obligor rating scale, some of the requirements can be relaxed. As proceeds from Guidelines on Validation (2006), in such a case there is no need for full compliance in the definition of default, and consequently for the calibration of either $L$ or the distance-to-default. Where a bank is intending to use the Merton's model as a direct method for estimating PD for obligors, supervisors will require a time series of appropriately defined defaults (long-run average default rate) with which either $L$ or the distance-to-default will be calibrated.

\subsection{Compliance with the data requirements}

Data must be representative for building the model (point c, paragraph 30, Part 4, Annex VII, CRD). Given that there is just one obligor is this method of assignment/estimation, and usually uninterrupted capture of market value of equity in recent years, the representativeness of the data primarily relates to the population from which the long-run average default rate is calculated and with which the model of PD estimates is calibrated. The representativeness of the population from which the empirical data on defaults is taken is important when the bank is using the Merton's model as a direct method for estimating PD. In general this means that empirical data can only be obtained from a population on which this model can be used. If the model is being used solely as a method for assigning obligors, the requirement for data representativeness can be relaxed. In the latter case the data for the long-run average default rate is of no relevance.

The time series of the data (the observation period) is also subject to minimum requirements. For the estimation of PD the length of the underlying historical observation period used shall be at least five years for at least one source (paragraph 66, Part 4, Annex VII, CRD). This means that, theoretically, direct estimates of PD from the Merton's model (this applies to all three methods discussed here) shall be made on five years of data on daily market value of equity and debt. In addition, this holds true only if all other assumptions of the model are valid. However, several questions arise with respect to the use of historical observation period, i.e. the time series of daily/quarterly/yearly market 
value of equity in the Merton's model. Is it compliant with the CRD that a bank is using estimates of PD based on one year of the data on daily market value of equity, while the $L /$ distance-to-default were calibrated with five years historical observation period on defaults? The answer might be affirmative as understood by the term "for at least one source". What time series of daily/monthly/yearly market value is sufficient? To what extent these requirements apply if the model was used solely as an assignment method?

\subsection{Distribution of natural logarithms of returns}

The Merton's model assumes a normal distribution for the natural logarithms of returns on the companys assets $\ln \left(A_{t} / A_{t-1}\right)$. This assumption often proves to be invalid. In particular this applies to the distribution of the daily market values of assets, estimated from daily market values of equity. This usually has a sharper peak, with comparatively greater density in the tails of the distribution. This does not apply solely to marginal, low-liquidity markets such as Slovenias, but also to more established markets. Some authors suggest that natural logarithms of returns are described by other distributions, such as those from the family of generalized hyperbolic distributions (e.g. Eberlein and Prause, $1998^{8}$ ). For supervisors it is important whether the estimation of PD takes into consideration statistically significant deviations in the distributions of returns from the normal, how such obligors are treated, and, in general, how this deficiency is eliminated or mitigated. Such a potentially erroneous assumption about the distribution of daily natural logarithms of returns can again be corrected by means of calibration of the distance-todefault or of $L$, which the MKMV method does when calculating the Expected Default Frequency. Such calibration or another correction is vital when the model is being used for estimating PD and for calculating capital requirements in the IRB approach.

\subsection{Constant return $\mu_{A}$ vs. risk-free interest rate $r$}

As stated, PD is estimated on the basis of (2.8), where either $\mu_{A}=r$ or $\mu_{A}$ is estimated on the basis of (3.4), or directly from (3.7). Inaccuracy in the estimated PD values can derive from the differences between $\mu_{A}$ and $r$, as $\mu_{A} \in \mathbb{R}$ (namely $\left.\mu_{A} \in \mathbb{R}_{+}\right)^{9}$, while $r$ is constant. Specifically, the calibration method does not provide for an estimate of $\mu_{A}$. This deficiency is of particular importance in the use of the calibration method and estimates of PD therefrom in the calculation of capital requirements in the IRB approach. The difference between $\mu_{A}$ and $r$ can also be a reason for the obligor being assigned a different credit rating, if it is assumed that the other values in (2.8) are unchanged. Therefore the calibration method is potentially less accurate both from the point of view of estimating PD and the point of view of assigning obligors to the obligor rating scale. If the minimum requirements of the CRD described above relate to the basic theoretical assumptions of the Merton's model, such as the definition of $L /$ distance-to-default with regard to empirical default, data representativeness, and the normal distribution of log returns, and apply to all three estimation methods, the difference in the estimation of PD

\footnotetext{
${ }^{8}$ Different asset value process is assumed, i. e. Lévy process.

${ }^{9}$ Hillegeist et al. (2004) stipulates that a negative $\mu_{A}$ is inconsistent with asset pricing theory. There is no limitation on the $\mu_{A}$ in this paper.
} 
or assignment resulting from the use of $\mu_{A}$ or $r$ relates to the different estimation methods of the model. Banks must demonstrate, insofar as they will use a calibration method, that it produces accurate estimates of PD or obligor ratings despite the use of $r$.

\section{Data}

Analysis of the performance of the three aforementioned methods was conducted on simulated and empirical data of Slovenian companies in SBI20. Simulation is used to imitate a real situation, i.e. the movement of market value of equity, and to derive general conclusions of the performance of used methods when estimating PD and assigning obligor grades. The empirical data is used to clearly present implementation and performance of mentioned methods on empirical data in general, and on Slovenian companies in particular, since to the author's knowledge this is the first attempt of estimating PDs with Merton (1974) model for Slovenian companies.

\subsection{Simulated data}

Simulated data were generated with the following properties. There were 5,000 simulations made of the geometric Brownian motion of daily market value of equity $E_{t}$, $t=(0, \ldots, 250)$, with $(2.3)$ and the following conditions:

- the parameter $\mu_{E}$ was set at 0.036 , and the value obtained was divided by 250 for adjustment to the daily value;

- the parameter $\sigma_{E}$ was randomly uniformly sampled in the interval between 0.1 and 1.0 , and the value obtained was divided by $\sqrt{250}$ for adjustment to the daily value;

- $E_{0}$ was always 1.0;

- the face value of debt $L$ was defined as follows: since the market value of debt $D_{t}$ plus the put option $\left(P_{t}\right)$ on the company's assets equals the discounted face value of debt $L$ by the risk-free rate, $L$ was

$$
L=\frac{E_{250}\left(D_{250}+P_{250}\right)^{\%}}{1-\left(D_{250}+P_{250}\right)^{\%}} e^{r} .
$$

In this paper the percentage $\left(D_{250}+P_{250}\right)^{\%}$ was randomly uniformly sampled in the interval between 0.1 and 0.8 .

\subsection{Empirical data: Selected companies from the SBI20}

The empirical data consisted of the market values of equity (shares) of companies listed on the Ljubljana Stock Exchange, and included in the SBI20 index, that had audited or unaudited consolidated balance sheets for 31 December 2005. These were Krka, Mercator, Petrol, Gorenje, Pivovarna Lako, Sava, Luka Koper, Intereuropa, Istrabenz, and Aerodrom Ljubljana. This decision was supported by the fact that these are the shares 
that are most heavily traded (daily) on the exchange, and the movement of their prices therefore approximates the movement of share prices on larger stock exchanges. In the Merton theoretical context this means that trading in equity proceeds uninterrupted. The time series of data from which $A_{t}, \mu_{A}$, and $\sigma_{A}$ were estimated, estimates of PD being made from these, encompasses all trading days in 2004 and 2005 ( $n=505$ days and no. of years =2).

\section{Results}

In this section the results are presented, separately for simulated and empirical data.

\subsection{Simulated data}

The relevant parameters were estimated on the basis of the simulated data using the methods described above for all 5,000 fictitious obligors. For each obligor a KolmogorovSmirnov test was conducted to verify the presumption of a normal distribution of returns on their assets. There was only one obligor in the simulated data whose distribution differed significantly from the normal at $95 \%$ confidence level. The described statistics (see Table 1) show that there are significant differences between the parameters estimated with the calibration method and the parameters estimated with the other two methods, under the given conditions of the simulation. The largest difference can be observed in the estimation of PD, where PDs from the calibration method are on the average one-third of the value of PDs estimated with MKMV or MLE methods. While the latter two produce estimates of PD in the interval of 0 to $100 \%$, PDs in the calibration method ranges between $0 \%$ and $30 \%$. On average $\sigma_{A}$ estimated with calibration method is $6 \%$ lower than if estimated with other two methods. Standard errors of $\mu_{A}$ are identical to $\sigma_{A}$ for a one year period, which is consistent with (3.5). Note that calibration method cannot produce the estimates of $\mu_{A}$ and standard errors of either $\mu_{A}$ or $\sigma_{A}$. The results imply the different level of calibration or accuracy in the estimates of PD between the methods, which ceteris paribus would generate differences in risk-weighted exposure amounts.

In accordance with the definition in paragraph 4 of Part 4 of Annex VII of the CRD, PD values calculated in this manner can be treated as credit ratings in a continuous rating scale, i.e. as ratings arranging obligors in terms of credit worthiness from best to worst. A Kendall $\tau_{b}$ (see Newson, 2001) correlation was used to verify if the Merton's model estimation methods are concordant or disconcordant in terms of assigning obligors grades. The correlation analysis shows that the MKMV and MLE methods indicates a perfect positive ordinal relationship in the assignment of obligor grades (a correlation of 1.0), while the correlation between PD from MKMV or MLE and calibration method is equal to 0.65 , also indicating a strong positive ordinal relationship, although not a perfect one. The latter implies different obligor grades assigned to the same obligors with calibration method or MKMV/MLE. 
Table 1: Estimation of parameters on the basis of the simulated data.*

\begin{tabular}{|c|c|c|c|c|c|}
\hline parameter & estimation method & mean & s.d. & minimum & maximum \\
\hline \multirow[t]{3}{*}{$A_{250}$} & calibration & 2.202 & 1.919 & 0.067 & 42.786 \\
\hline & MKMV & 2.199 & 1.930 & 0.067 & 43.705 \\
\hline & MLE & 2.199 & 1.930 & 0.067 & 43.707 \\
\hline \multirow[t]{2}{*}{$\mu_{A}$} & MKMV & -0.019 & 0.389 & -2.395 & 1.901 \\
\hline & MLE & -0.020 & 0.389 & -2.400 & 1.896 \\
\hline \multirow[t]{3}{*}{$\sigma_{A}$} & calibration & 0.313 & 0.193 & 0.023 & 0.940 \\
\hline & MKMV & 0.333 & 0.208 & 0.021 & 0.993 \\
\hline & MLE & 0.331 & 0.207 & 0.021 & 0.989 \\
\hline \multirow[t]{2}{*}{ s.e. $\left(\mu_{A}\right)$} & MKMV & 0.333 & 0.208 & 0.021 & 0.993 \\
\hline & MLE & 0.331 & 0.207 & 0.021 & 0.989 \\
\hline \multirow[t]{2}{*}{ s.e. $\left(\sigma_{A}\right)$} & MKMV & 0.235 & 0.147 & 0.015 & 0.702 \\
\hline & MLE & 0.234 & 0.146 & 0.015 & 0.697 \\
\hline \multirow[t]{3}{*}{ PD } & calibration & 3.229 & 5.241 & 0.000 & 30.261 \\
\hline & MKMV & 10.270 & 20.276 & 0.000 & 99.528 \\
\hline & MLE & 10.239 & 20.273 & 0.000 & 99.539 \\
\hline
\end{tabular}

${ }^{*}$ In the first column estimated parameters are listed: $A_{250}$ is the market value of assets in $t=250 ; \mu_{A}$ and $\sigma_{A}$ are the drift and the standard deviation, respectively, of natural logarithms of asset returns, with their standard errors s.e. $\left(\mu_{A}\right)$ and s.e. $\left(\sigma_{A}\right)$; PD is the estimate of probability of default in \%. Estimation methods are listed in the second column, where standard errors of $\mu_{A}$ and $\sigma_{A}$ under the MKMV method are estimated with (3.5) and (3.6), respectively. For PD estimates, $r$ is used for calibration method and $\mu_{A}$ for other two methods in (2.8). In all other columns the statistics are presented.

In order to analyse the influences on differences in estimates of PD, a simple linear regression analysis was conducted (see Table 2 ). The estimated parameters $\hat{A}, \hat{\sigma}_{A}, \hat{\mu}_{A}$, PD etc. were treated as random variables for this purpose with $n=5000$ observations. The normalization of the variables was used to equalize the measurement scales and to fullfill the presumption of the normal distribution. The dependent variable was the normalized absolute difference in the estimates of PD between MKMV and calibration method, denoted $\left|P D^{M K M V}-P D^{c a l}\right|$. The inclusion of explanatory variables was partially based on observations of Crosbie and Bohn (2003), who stipulates that (3.1) holds only instantaneously, and that the market leverage is too volatile to provide accurate estimates of $\sigma_{A}$. They found bias in PD estimates when sharp increase/decline of leverage is noticed. Following these observations, two explanatory variables were included:

- the normalized volatility of leverage in the time series, denoted $\sigma_{D / A}$, and estimated as 


$$
\begin{aligned}
\sigma_{D / A} & =\sqrt{\frac{1}{n} \sum\left(D_{t} / A_{t}-\overline{D / A}\right)^{2}}, \\
\overline{D / A} & =\frac{1}{n} \sum_{t=0}^{n}\left(D_{t} / A_{t}\right)
\end{aligned}
$$

- the normalized absolute difference between the initial and final leverages, i.e. the leverage in $t=0$ and $t=250$, denoted $\left|D_{0} / A_{0}-D_{250} / A_{250}\right|$;

In addition, the following variables were included:

- the normalized standard deviation of the natural logarithms of equity returns, denoted $\sigma_{E}$, since this is the main driver of both asset and leverage volatility;

- the normalized absolute difference between drift estimated with MKMV method and risk-free interest rate, denoted $\left|\mu_{A}-r\right|$, based on the arguments in subsection 4.4

- the normalized leverage in $t=250$, denoted $D_{250} / A_{250}$;

Table 2: Influences on differences in estimates of PD.*

\begin{tabular}{l|rrrr}
\hline variables & $\beta$ & $\mathrm{t}$ & sig. & tolerance \\
\hline$\sigma_{D / A}$ & 0.186 & 17.041 & 0.000 & 0.252 \\
\hline$\left|\frac{D_{0}}{A_{0}}-\frac{D_{250}}{A_{250}}\right|$ & 0.209 & 18.964 & 0.000 & 0.246 \\
\hline$\sigma_{E}$ & 0.467 & 56.508 & 0.000 & 0.440 \\
\hline$\left|\mu_{A}-r\right|$ & 0.164 & 15.413 & 0.000 & 0.265 \\
\hline$D_{250} / A_{250}$ & 0.274 & 38.700 & 0.000 & 0.600 \\
\hline
\end{tabular}

${ }^{*} \mathrm{n}=5000, r^{2}=.85$, the dependant variable is $\left|P D^{M K M V}-P D^{c a l}\right|, \beta$ are standardized regression coefficients, $t$ is the value from $t$ distribution, sig. is statistical significance of $\beta$, and tolerance is the variance of the variable that is not explained by others in the model.

In this simple model the explained variance is 0.85 , and all $\beta$ s are positive and significantly different from zero. The main driver of the differencies in PD estimates between MKMV/MLE and calibration methods is the standard deviaton of natural logarithm of equity returns, followed by the leverage in $t=250$. This means that the larger values of these quantities generate larger differencies in PD estimates. Under the given setting of the simulation, the result of the regression analysis on the simulated data is intuitive, and in general confirms the findings of other authors that the calibration method does not 
satisfactorily take into consideration the dynamics of market value of equity in combination with the dynamics of leverage in the time series. It is worth emphasizing that there is considerable correlation among the explanatory variables, indicated by the variance in individual variables that is not explained by the other variables in the model (tolerance).

The results on the simulated data show that in the context of the IRB approach it is possible to determine both the level of difference in the assignment of obligors to the obligor rating scale (Kendall's $\tau_{b}$ ) and also the different calibration (or accuracy) of direct estimates of PD between the calibration method and the MKMV/MLE methods.

\subsection{Empirical data}

Table 3 (in the Appendix I) illustrates the values that served for the estimation of the relevant parameters and the estimated parameters at the final day of 2005. KolmogorovSmirnov test reveals that the presumption of the normal distribution of natural logarithms of returns on assets could be rejected for all companies at $95 \%$ confidence level. The estimates of the parameters confirm the findings on the simulated data. The differences are observed mainly in the estimates of PD, while the estimates of the other parameters are similar regardless of the estimation method. The reason for the similarity in the estimated parameters is low volatility of the market value of assets and leverage, as indicated by the regression analysis.

Table 3 shows that the one-year $\mathrm{PD}$, which is also a requirement of the $\mathrm{CRD}$, is smaller than $0.03 \%$ for all companies. This percentage is the minimum possible PD for companies under the CRD, despite the estimation including the total book liabilities. Part of the reason for the extremely low PD could be found in the distributions of returns, which are not normal. Distributions have greater density in the tails, but a comparatively thinner tail in the normal distribution cannot describe this. Crosbie and Bohn (2003) also cite this fact, adding that a distance-to-default of 4 standard deviations, on the basis of mapping to empirical data on defaults, entails a PD of $1 \%$, while the theoretical PD from the normal distribution is practically zero. Such effect is not analyzed in this paper.

\section{Conclusion}

The use of the traditional 1974 Merton structural model for calculating PD in light of the minimum requirements of the IRB approach was illustrated in this paper. Differences in the estimates of PD made using three estimation methods were analyzed, namely the calibration method, the MKMV method, and the MLE method. The results are based on simulated data and on empirical data from certain Slovenian companies in the SBI20. The structural model is not directly useful for estimating PD for the purpose of calculating capital requirements in the IRB approach until the estimates have been calibrated using empirical data on defaults. And conversely, only when the data on defaults for listed companies is available and back-testing has been facilitated can such models be used in the IRB approach. The empirical data on defaults is used to eliminate the deficiencies in the model such as the simple debt structure and the assumption of the normal distribution of natural logarithms of returns on assets. In addition, in the IRB approach the minimum requirements outside the model must also be fulfilled. These are the definitions of default, 
which must be in accordance with the definition in the CRD, the representativeness of the data based on which $L$ or the distance-to-default is (potentially) calibrated, a sufficient time series of data and, not least, sufficient predictive ability and discriminatory power.

The results also indicate that differences in the estimated PD values can be the result of the estimation methods. Differences in estimated PD were observed between the calibration method and the MKMV/MLE methods. The calibration method is deficient in two aspects. The first is that, as a result of the estimation of $\sigma_{A}$ at a single point of time, the calibration method does not take into consideration sufficient equity volatility in combination with the dynamics of leverage in the time series from which it is estimated by both the MKMV and the MLE methods. The second deficiency is correlated with the first, and relates to drift $\mu_{A}$, which the calibration method does not estimate, the risk-free interest rate $r$ (commonly) being used instead.

As illustrated in the paper, the stipulated minimum requirements of the CRD can be relaxed if the model is being used to assign obligors to an obligor rating scale. Several pieces of research confirm that the Merton's model has good discriminatory power. Vassalou and Xing (2004) state that the PD from the Merton's model is actually a default likelihood indicator, and not a direct measure of PD. Bharath and Shumway (2008) show that there is high correlation between the credit ratings by Moodys and the estimates of PD as estimated in their paper based on the Merton's model. This is an indication of the potentially satisfactory discriminatory power of the Merton's model, which is one of the key items for the acceptance of assignment methods by supervisors (see for example Guidelines for Validation, 2006). However, there are differences in assignment as a result of the different estimation methods. The Kendall $\tau_{b}$ correlation shows deviation from a perfect positive ordinal relation in assigning obligor grades between calibration and MKMV/MLE methods, when the rating scale is highly granulated. In evaluating rating systems and/or methods for estimating PD, the final and most important criterion is discriminatory power and/or predictive ability (shown via back-testing). It is therefore vital that there is empirical, valid and correctly defined data on defaults, irrespective of the model or the mechanical method of assignment and/or estimation.

\section{Acknowledgements}

The author thanks Paola Re Fiorentin for the extensive contribution to the paper and Dirk Tasche for valuable comments. The opinions expressed in this paper are those of the author and do not necessarily reflect views of Bank of Slovenia.

\section{References}

[1] Altman, E. (2006): Default Recovery Rates and LGD in Credit Risk Modeling and Practice: An Updated Review of the Literature and Empirical Evidence. Available on http://www.defaultrisk.com/pp_recov_53.htm

[2] Basel Committee on Banking Supervision, 2004. An Explanatory Note on the Basel II IRB Risk Weight Functions. Basel: Bank for International Settlements. Available on bis.org. 
[3] Bharath, T. S. and Shumway, T. (2008): Forecasting default with the Merton distance to default model. The Rewiev of Financial Studies, 21, 1339-1369.

[4] Black, F. and Scholes, M. (1973): The pricing of option and corporate liabilities. The Journal of Political Economy, 81, 637-654.

[5] Bluhm, C., Overbeck, L., and Wagner, C. (2003): An Introduction to Credit Risk Modeling. London: Chapman\&Hall/CRC Press.

[6] Bruche, M. (2005): Estimating Structural Bond Pricing Models via Simulated Maximum Likelihood. Available on http://www.defaultrisk.com/pp_model_96.htm.

[7] Campbell, Y.J., Lo, W.A., and MacKinlay, A.C. (1996): The Econometrics of Financial Markets. Princeton: Princeton University Press.

[8] Committee of European Banking Supervisors (2006): Guidelines on the Implementation, Validation and Assessment of Advanced Measurement (AMA) and Internal Ratings Based (IRB) Approaches. London: Committee of European Banking Supervisors. Available on http://www.c-ebs.org.

[9] Crosbie, J.P. and Bohn, R.J. (2003): Modeling Default Risk. Moody's KMV. Available on www.defaultrisk.com/pp_model_35.htm.

[10] Davydenko, A.S. (2005): When Do Firms Default? A Study of the Default Boundary. Available on www.defaultrisk.com/pp_score_51.htm.

[11] Directive 2006/48/EC of the European Parliament and of the Council (2006).

[12] Duan, J.C. (1994): Maximum Likelihood Estimation using Price data of the derivative contract. Mathematical Finance, 4, 155-167. A correction published in Mathematical Finance, 10, 461-462.

[13] Duan, J.C., Gauthier, G., and Simonato, J.G. (2004): On the Equivalence of the KMV and Maximum Likelihood Methods for Structural Credit Risk Models. Available on www.defaultrisk.com/pp_model_97.htm.

[14] Duffie, D., Saita, L., and Wang, K. (2005): Multi-period corporate failure prediction with stochastic covariates. Journal of Financial Economics, 83, 635665.

[15] Eberlein, U. and Prause, K. (1998): The Generalised Hyperbolic Model: Financial Derivatives and Risk Measures. Working paper Nr. 56, University of Freiburg.

[16] Elizalde, A. (2005): Credit Risk Models II: Structural Models. Available on www.defaultrisk.com/pp_model_86.htm

[17] Ericsson, J. and Reneby, J. (2005): Estimating structural bond pricing models. Journal of Business, 78, 707-736.

[18] Gordy, M. (2003): A Risk-Factor Model foundation for Ratings-Based Bank Capital Rules. Journal of Financial Intermediation, 12, 199-232. 
[19] Hillegeist, A.S., Keating, K E., Cram, P.D., and Lundstedt, G.K. (2004): Assessing the probability of bankruptcy. Review of Accounting Studies, 9, 5-34.

[20] Merton, C.R. (1974): On the pricing of corporate debt: The risk structure of interest rates. Journal of Finance, 29, 449-470.

[21] Newson, R. (2001): Parameters behind non-parametric statistics: Kendalls $\tau_{a}$, Somers $D$ and median differences. The Stata Journal, 1, 1-20.

[22] Oesterreichische Nationalbank and Austrian Financial Market Authority (2004): Rating Models and Validation. Vienna. Available on www.oenb.at/en/img/rating_models_tcm16-22933.pdf.

[23] R Development Core Team (2009): R: A language and environment for statistical computing. R Foundation for Statistical Computing, Vienna, Austria. ISBN 3900051-07-0. Available on http://www.R-project.org.

[24] Vassalou, M. and Xing, Y. (2004): Default risk in equity returns. Journal of Finance, 59, 831-868.

\section{Appendix I}




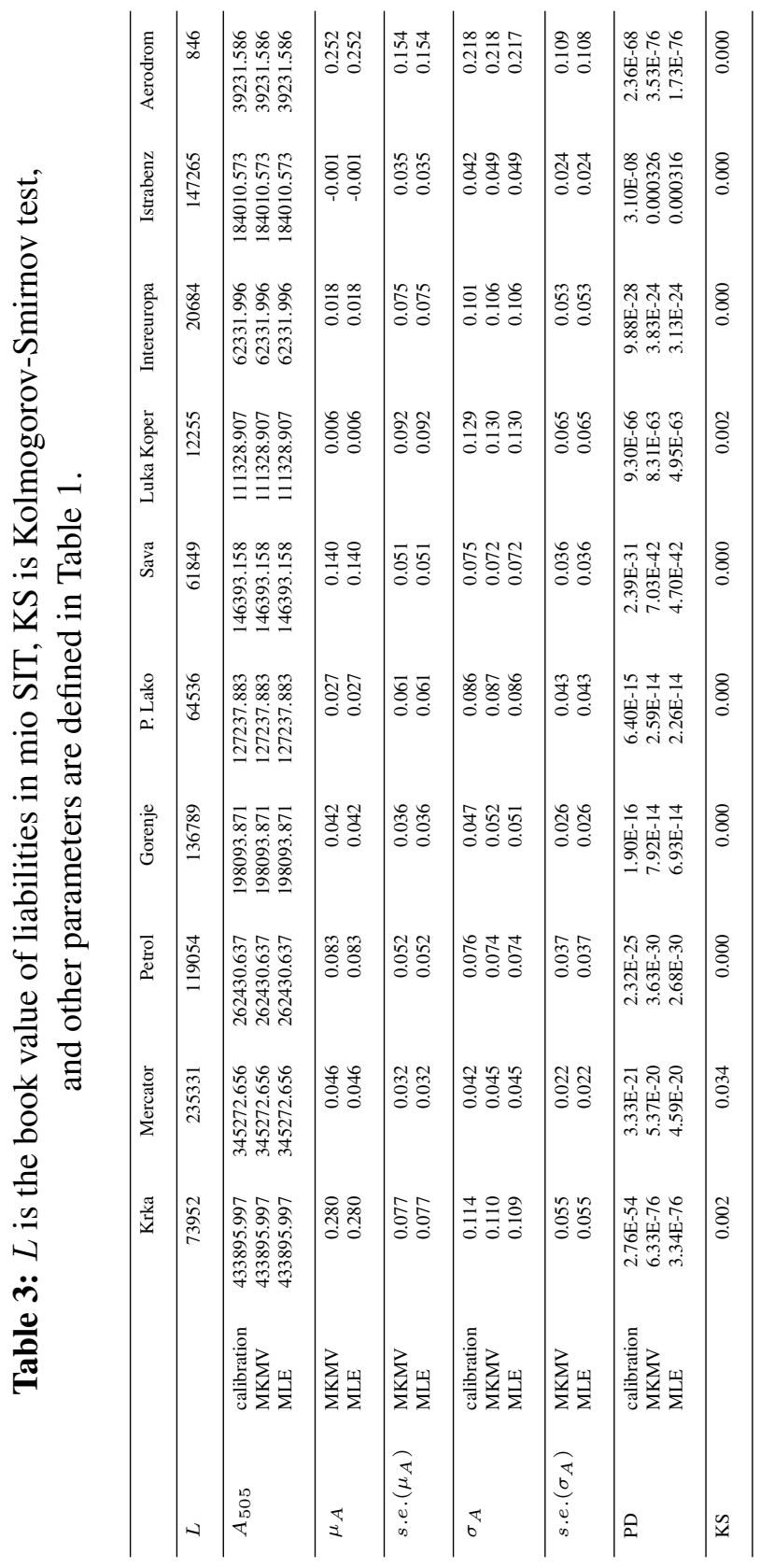

